Research Paper

\title{
Pretreatment of Cisplatin in Recipients Attenuates Post-Transplantation Pancreatitis in Murine Model
}

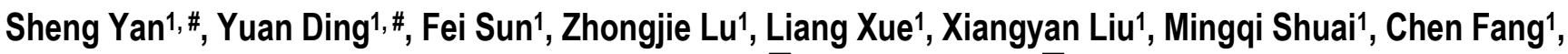 \\ Yan Wang', Hui Cheng', Lin Zhou', Ming H Zheng ${ }^{1,2,}$, Shusen Zheng ${ }^{1, 凶}$
}

1. Division of Hepatobiliary and Pancreatic Surgery; Key Laboratory of Combined Multi-organ Transplantation, Ministry of Public Health; and Key Laboratory of Organ Transplantation Zhejiang Province, First Affiliated Hospital, Zhejiang University School of Medicine, Hangzhou 310003, P.R. China.

2. Centre for Orthopaedic Research, School of Surgery, University of Western Australia, Western Australia, 6009, Australia.

\# These authors contributed equally to this paper.

$\square$ Corresponding author: Shu-Sen Zheng, MD, PhD, FACS, Key Laboratory of Combined Multi-organ Transplantation, Ministry of Public Health, First Affiliated Hospital, Zhejiang University School of Medicine, Hangzhou 310003, China (Tel: 86-571-87236570; Fax: 86-571-87236884; Email: shusenzheng@zju.edu.cn); Or W/Prof. Minghao. Zheng, Centre for Orthopaedic Research, University of Western Australia, M Block, QEII Medical Centre, Monash Avenue, Nedlands, Western Australia 6009, Australia. Tel: 61-8-9346-4050; E-mail: minghao.zheng@uwa.edu.au.

(c) Ivyspring International Publisher. This is an open-access article distributed under the terms of the Creative Commons License (http://creativecommons.org/ licenses/by-nc-nd/3.0/). Reproduction is permitted for personal, noncommercial use, provided that the article is in whole, unmodified, and properly cited.

Received: 2011.10.18; Accepted: 2011.12.21; Published: 2012.02.01

\begin{abstract}
Pancreas transplantation is the definite treatment for type I diabetes that enables the achievement of long-term normoglycemia and insulin independence. However Post-Transplantation Pancreatitis (PTP) due to ischemia reperfusion (IR) injury and preservation is a major complication in pancreas transplantation. Owning the potential anti-inflammatory effect of Cisplatin (Cis) in liver IR injury, we have examined if Cis could attenuate PTP using a murine model. We found that $\mathrm{Cis}$ is able to prevent inflammatory response in PTP. Pretreatment of Cis in recipient mice reduce the impairments of the grafts and hyperamylasimea in the recipients. We documented that the protective mechanism of $\mathrm{Cis}$ in PTP involves improvement of microcirculation, reduction of the mononuclear cellular infiltration and apoptosis, suppression of inflammatory cytokine-cascade and inhibition of translocation of high-motility group box protein-I (HMGB-I) from nucleus to cytoplasm. In short, our study demonstrated that pretreatment of $\mathrm{Cis}$ in recipients may reduce the onset of PTP in pancreas transplantation.
\end{abstract}

Key words: Cisplatin, Pancreatitis, Ischemia reperfusion injury, High-motility group box protein-1, Intravital fluorescence microscopy.

\section{Introduction}

Pancreas transplantation is the definite treatment for type 1 diabetes that enables the achievement of long-term normoglycemia and insulin independence. The technique had evolved many years since the first clinical transplant in 1966, by the team of Dr. Kelly and Dr. Lillehei [1,2]. However, a variety of post-transplant complications, such as immunological rejection, recurrent autoimmunity and inflammation has been reported. Among of them PTP remains the main cause of intra-abdominal abscess, fatal infection and other motilities [3,4]. Unlike acute bilogenic or alcoholic pancreatitis induced by exocrine occlusion or chemical stimuli, organ preservation and ische$\mathrm{mia} /$ reperfusion (IR) injury is the primary cause for 
PTP.

The reoxygenation of the hypoxic pancreatic acini after IR triggers the inflammatory mediators and microcirculatory disturbance [5]. The physiological alterations of PTP resemble general pancreatitis, but are featured by the aggressive progression and poor respond to treatment $[6,7]$. Several factors, such immunesuppression, transplant site, rejection and septic tendency, have been showed to contribute to the pathology of the PTP [8]. Currently there are many attempts in searching potential agents that are able to mitigate PTP.

Cisplatin (CIS) has been used as chemotherapy drug in the treatment of cancers for more than 30 years [9]. It causes conformational changes in the double helix of DNA that contains binding site of HMGB-1 (high-motility group box protein 1) [9]. Crosslink of DNA by Cis results in interfering of mitosis and activating apoptosis of target cell. Interestingly, Recent reports has indicated that Cis at optimal low does is shown to have a protective effect for ischemia reperfusion injury in murine liver and hepatocytes subjected to hypoxic exposure [9]. The mechanism was partially due to the inhibition of HMGB-1 releasing from the nucleus of the hypoxic cells [9].

The protective effects of Cis in prevention of liver IR injury was intriguing, thus provoking our interests to examine its role in PTP. We modified a murine model of pancreas transplantation with preserved exocrine drainage, that can mimic the PT in human[6]. Using this model, we studied the protective effects of Cis on graft pancreatitis. We hypothesize that Cis might attenuate the graft impairments by inhibiting the local inflammation response in graft after PTP.

\section{Materials and methods}

\section{Animals}

Male Balb/c mice aged from 8 to 12 weeks, obtained from Shanghai Animal Center (Chinese Academy of Science, Shanghai, China), were used as size matched donor and recipient pairs. The mice were housed in standard pathogen free condition and with free access to standard rodent food and water. All animal experiments were approved by the Animal Care Committee of Zhejiang University in accordance with the Principles of Laboratory Animal Care.

\section{Experiment design and surgical procedure}

Donor operation: Donor pancreas were harvested from Balb/C mice by non-touch technique [6], the pancreatic head ligated but the main pancreatic duct open by catheterization. The grafts were stored in UW (University of Wisconsin solution) solution at $4^{\circ} \mathrm{C}$ for $16 \mathrm{~h}$ (Cold ischemia time of graft is 16 hours).

Recipient operation: The optimization of final dose selection and the time point for Cis pretreatment is based on previous study by Tsung's group. $12 \mathrm{~h}$ prior to recipient operation, mice were injected intraperitoneally $0.5 \mathrm{ml}$ PBS (Control group) or Cis at three different dosages $(0.1 \mathrm{mg} / \mathrm{kg}, 1 \mathrm{mg} / \mathrm{kg}$, and 10 $\mathrm{mg} / \mathrm{kg}$; PBS was used for dissolve Cis. $\mathrm{n}=6$ respectively). Graft was implanted in the recipient's cervical region by connecting the portal vein to the external jugular vein and the donor aortic segment to the carotid artery by cuff technique[6, 10].

Experiments: Six animals were used in each group. Specimens were harvested after 6 hours of transplantation and cut into small pieces for histopathology, immunohistochemistry, Tunel assay and Western blotting, respectively; Serum was collected for amylase assay. Graft microcirculation was analyzed by intravital fluorescence microscopy (IVM) $2 \mathrm{~h}$ after reperfusion. Flow chart and the experimental design were shown in Fig. 1.

\section{Agents and Antibodies}

Cis was purchased from Sigma Inc., All the antibodies used in this study are listed below: Apop-Tag Peroxidase In Situ Apoptosis Detection Kit (cat\#S7100; Chemicon International Inc., Billerica, MA, USA), Anti-IL-1-beta antibody (cat\#ab9722,abcam,UK), Anti-IL6 antibody (cat\#ab6672, abcam,UK), Anti-TNF alpha antibody (cat\#ab6671, abcam,UK), Mouse Myeloperoxidase /MPO Antibody(cat\# 392105, R\&D Systems, Inc). Fuorescein-isothiocyanate-labeled dextran (FITCdextran) was obtained from Sigma Chemical Co., St. Louis, MO, USA.

\section{Histopathology}

Specimens were fixed in $10 \%$ formaldehyde over 24 hours, dehydrated and embedded in paraffin. $5 \mu \mathrm{m}$ sections were made and stained with hematoxylin and eosin (H\&E). The sections were scored with Schmidt's method for inflammatory infiltration, interstitial edema, acinar cell necrosis and hemorrhage-fat necrosis by independent pathologists [11].

\section{Immunohistochemistry}

Specimens from histopathology were sectioned for immunohistochemistry. In brief, sections $(5 \mu \mathrm{m})$ were de-waxed and rehydration, pancreas sections were put in $3 \% \mathrm{H}_{2} \mathrm{O}_{2}$ for 15 minutes to block endogenous peroxidase and then were boiled in sodium citrate buffer $\mathrm{pH} 6.0$ for 15 minutes. After incubated in 
$5 \%$ fetal serum phosphate buffered saline for 30 minutes at room temperature, the sections were added with MPO antibodies at 1:50 dilution at $4^{\circ} \mathrm{C}$ overnight and in goat-anti rabbit secondary antibody at 1:500 dilution for 30 minutes at room temperature. Diaminobenzidine were applied for 3 minutes and then the sections were counterstained with hematoxylin.

To improve the antigen retrieval, sections were boiled in sodium citrate buffer $\mathrm{pH} 6.0$ for $15 \mathrm{~min}$ and incubated in 5\% fetal serum phosphate buffered saline for $30 \mathrm{~min}$ at room temperature, and then incubated in HMGB-1 antibody at 1:200 dilution at $4^{\circ} \mathrm{C}$ overnight. At the second day Alexa-fluor donkey anti-rabbit IgG (Invitrogen) were applied to the section at 1:200 dilutions for 30minutes at room temperature. After Dapi dye, the sections were washed three times with PBS and coverslipped with glycerol. Images were obtained by LEICA CTR 5500 (LEICA) and analyzed by image-pro plus 6.0 software.

\section{Tunel assay}

The paraffin sections of specimens were stained for apoptotic cells by the Tunel assay using a commercially available kit (Apop-Tag Peroxidase In Situ Apoptosis Detection Kit S7100; Chemicon International Inc., Billerica, MA, USA). The Tunel assay was performed according to the manufacturer's protocol. The results were presented as the mean number of Tunel-positive cells per high power field.

\section{Amylase assay}

Six hours after reperfusion, blood samples were obtained from retrobulbar venous plexus and centrifuged at $3000 \mathrm{r} / \mathrm{m}$ for 15 minutes and stored at $-80^{\circ} \mathrm{C}$ before analysis. The amylase level in serum was measured using an Automated Chemical Analyzer (7600; Hitachi, Tokyo, Japan).

\section{Western Blotting}

Mice from each group were sacrificed 6 hours after reperfusion and half of pancreatic grafts were homogenized in lysis buffer, sonicated and centrifuged at 5,000 rpm. The extracts contain equal volume and equal protein concentration were separated by $12 \%$ sodium dodecyl sulfate-polyacrylamide gel electrophoresis (SDS-PAGE) and transferred to nitrocellulose membranes. The membrane was blocked with $5 \%$ fetal serum-Tris-buffered saline (TBS) with $0.1 \%$ Tween 20 (TBS-T) at room temperature for 2 hours. Then membranes were incubated in rabbit-anti mice polyclonal HMGB-1, IL-1, IL-6 or TNF-alpha (dilution $=1: 1000$ ) at $4^{\circ} \mathrm{C}$ overnight. After three washes of TBS-T the membranes were then incubated with goat anti-rabbit secondary antibody at (dilution=1:1500) at room temperature for 2 hours. Results were obtained by KODAK image station 2000R and analyzed by image-pro plus 6.0 software.

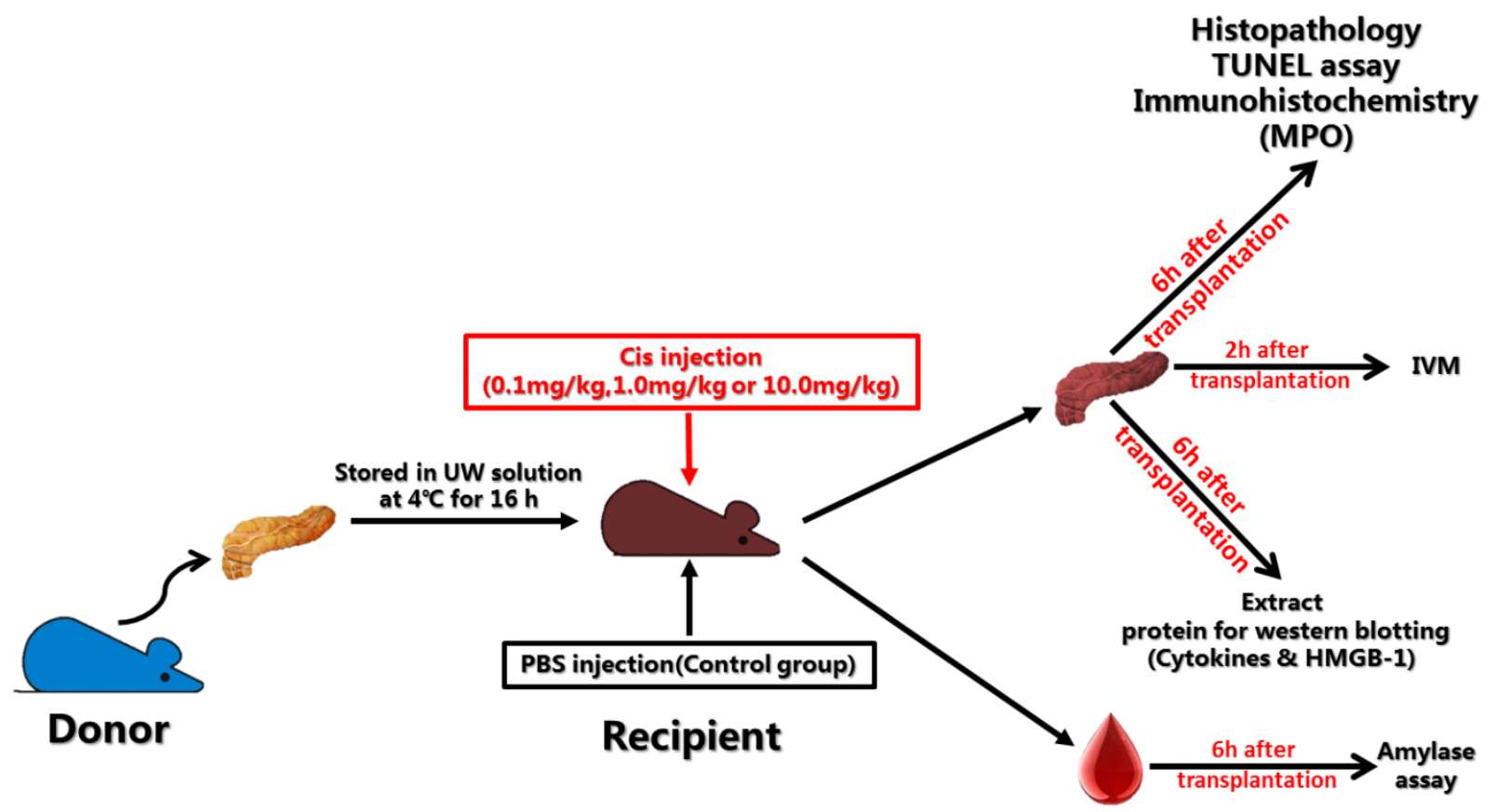

Fig. I. Flow chart and experiment design of pancreases transplantation. 


\section{Intravital fluorescence microscopy (IVM)}

IVM was used to analyze graft microcirculation injury by means of functional capillary density (FCD) and capillary diameters (CD). Stationary of microcirculatory changes was conducted by quantification of functional capillary density (FCD) in ten randomly selected areas. Measurements were performed $120 \mathrm{~min}$ after reperfusion (total time of ischemia-reperfusion for IVM $=16 \mathrm{~h}$ cold ischemia time $+2 \mathrm{~h}$ reperfusion time). FCD, the length of red blood cell perfused capillaries per defined area (centimeters per square centimeter), was at the vessel wall for at least $30 \mathrm{~s}$. [12]. In brief, recipient mice were injected with Cis $(1 \mathrm{mg} / \mathrm{kg})$ or PBS $12 \mathrm{~h}$ before transplantation. Two hours after transplantation (total ischemia-perfusion time is 16 hours), mice were placed in a prone position, and the pancreatic graft was placed on a slide in warm saline at $37^{\circ} \mathrm{C}$. Blood perfusion within individual micro vessels was examined after intravenous injection of fluorescein-isothiocyanate-labeled dextran (FITCdextran) at a dose of (i.v. $0.3 \mathrm{ml}, 4 \mathrm{mg} / \mathrm{ml}$ ) [12]. An equilibration period of 5 minutes was allowed before starting the microscopic observation. IVM was performed using a modified Olympus microscope (IX81WI; Olympus Optical Corporation GmbH, Hamburg, Germany). Filter blocks for fluorescein (excitation 465-495 nm, emission $>515 \mathrm{~nm}$ ) and rhodamine (excitation 510-560 nm, emission $>590 \mathrm{~nm}$ ) were used for epi-illumination (Olympus), and the images were acquired using KAPPA Image Base software. Quantitative image analysis was performed with PicEd Cora (JOMESA, Munich, Germany) software. (More detailed technical information please refer to ref. [12])

\section{Statistical analyses}

All statistics have been calculated using the prism 5.01 software package (GraphPad Software 5.0, USA). All data are presented as mean values \pm standard error of the mean (SEM). Analysis of variance (ANOVA) and $\mathrm{T}$ test of independent means were used for statistical analysis. Data were considered significant at a level of $\mathrm{p}<0.05$.

\section{Results}

\section{Cis pretreatment attenuates PTP in Mice}

Recipient Balb/c mice were given injections of PBS or Cis 12 hours before transplantation. Six hours after reperfusion (total time of ischemia-reperfusion is $16 \mathrm{~h}$ cold ischemia time plus $6 \mathrm{~h}$ reperfusion time), specimens were harvested and were subjected for histological examination. Semi quantitative histological scoring of interstitial edema, inflammation, acinar necrosis and hemorrhage-fat necrosis were measured using the method described by Schmidt et al [11]. The results showed that pretreatment of Cis in recipients display a reduction of local inflammation. The reduction of mononuclear cell infiltrates in parenchyma and perivascular regions of the grafts was very obvious when compared to the PBS control (Fig.2A-D). Semi quantitative assessment showed that the suppression of inflammation is in a dose dependent manner (Fig.2E). It is noteworthy that acinar necrosis and hemorrhage-fat necrosis were not observed in both PBS and Cis groups.

\section{Cis protects pancreas acinar cell from apop- tosis after transplantation.}

Next, we examine if Cis prevents acinar cell apoptosis in pancreatic grafts after transplantation. Using Tunel assay we found that pretreatment of Cis results in reduction of numbers of apoptotic acinar cells (Fig. 3). It appears that only Cis at a dose over $1 \mathrm{mg} / \mathrm{kg}$ can achieve significant effect evidenced by the reduction of total cell counts of apoptotic cells per field. This result suggested that Cis at the optimal dose of $1 \mathrm{mg} / \mathrm{kg}$ is capable to protect acinar cell apoptosis (Fig. 3).

\section{Serum Amylase was marked reduced by pre- treatment of Cis}

Serum amylase levels are widely accepted as an indicator of acinar cell necrosis and subsequent pancreatic tissue injury in pancreatitis [14]. Although we did not observe obvious acina cell necrosis in both PBS and Cis treatment groups, serum amylase may be changed due to the onset of PTP. To this end, we used serum amylase as a surrogate marker for protective effect in acinar cells. To examine if Cis has protective effect on acinar cells after transplantation, the levels of amylase in sera at 6 hours after transplantation were measured. The result showed that in comparison with the PBS control group, the serum amylase was reduced by pretreatment of Cis at a dose of $1 \mathrm{mg} / \mathrm{kg}$. However, Cis at doses of 0.1 and $10 \mathrm{mg} / \mathrm{kg}$ failed to achieve a significant effect on the levels of amylase (Fig. 4). The failure of dose response may reflect to the sensitivity of the serum amylase assay. Nevertheless, taking together with the histological assessment and apoptosis assays, we concluded that Cis pretreatment can reduce the severity of the post-transplant pancreatitis by the protection of acinar cell death and inhibition of local inflammation of the graft.

\section{Cis reduces the impairments of microcircula- tion in grafts}

Survival of graft after transplantation depends 
on the restoration of microcirculation. To quantify the dynamics of microcirculation after transplantation, we have used IVM in real time to measure FCD (functional capillary density) and CD (capillary diameters) $2 \mathrm{~h}$ after transplantation (total ischemia time 16 hours plus reperfusion time 2 hours) in mice. Basic on the previous studies described above, we have chosen the optimal dose of $1 \mathrm{mg} / \mathrm{kg}$ of Cis for real time IVM experiments [12-15]. Our results showed that in the recipients receiving PBS as control, the cold preserved grafts for 16-hour preservation plus 2 hours

B

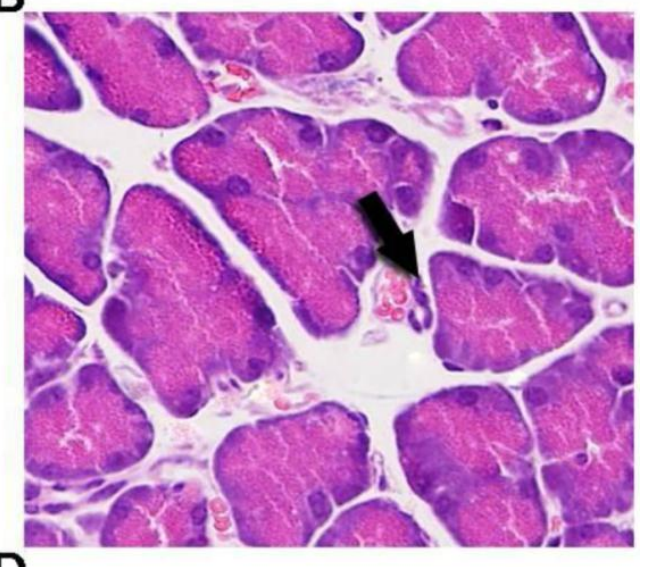

D

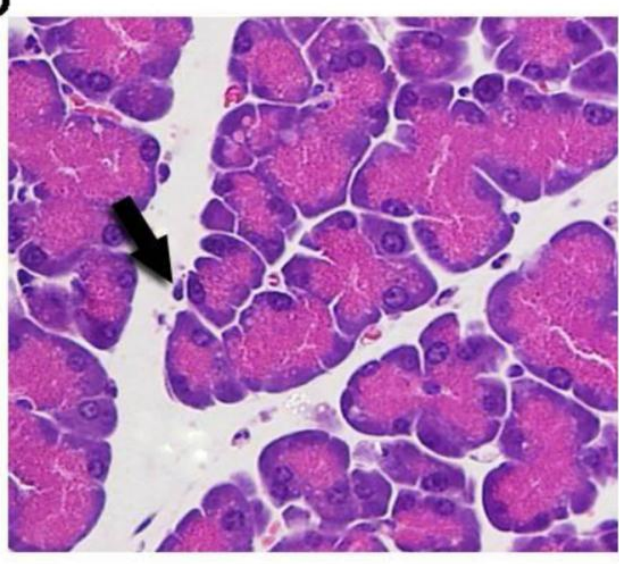

reperfusion after transplantation had relatively poor microcirculatory (Fig 5A). In contrast, pancreatic grafts with pretreatment of Cis under identical condition as to the control showed significant enrichment of the microcirculatory architecture compared with the PBS control group (Fig. 5B). Quantitative analysis of FCD and CD showed that while the CD remains unchanged (Fig. 5C), the FCD was significantly increased after treatment of Cis (Fig. 5D). Our results revealed that Cis may inhibit the occurrence of perfusion disorders in the early period of PTP.
A
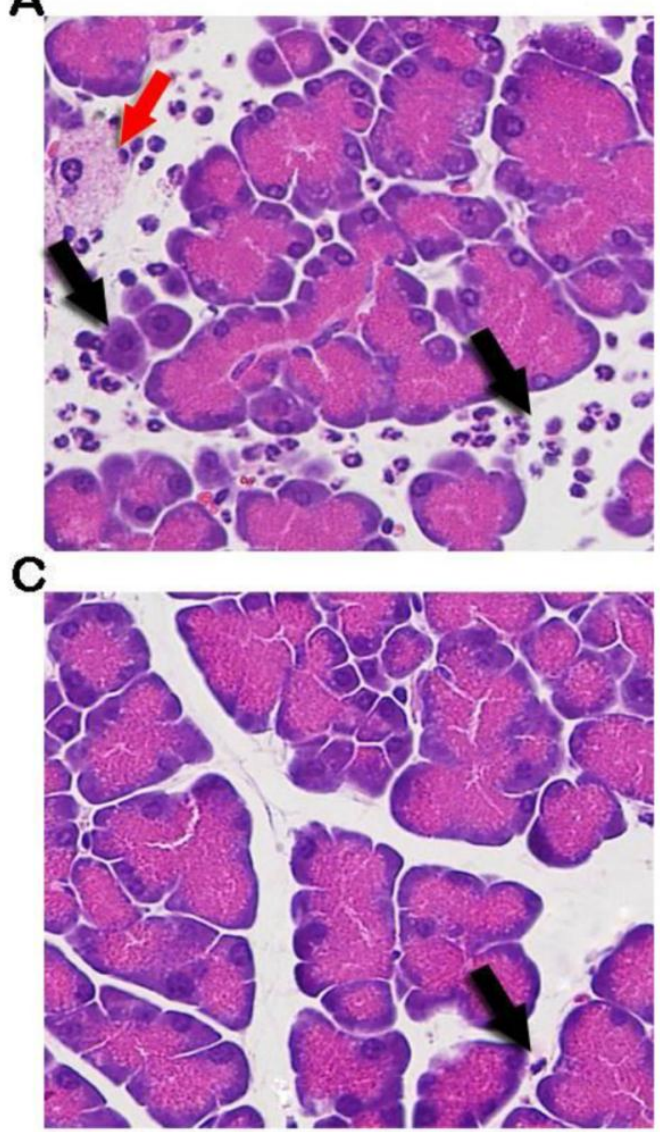

E

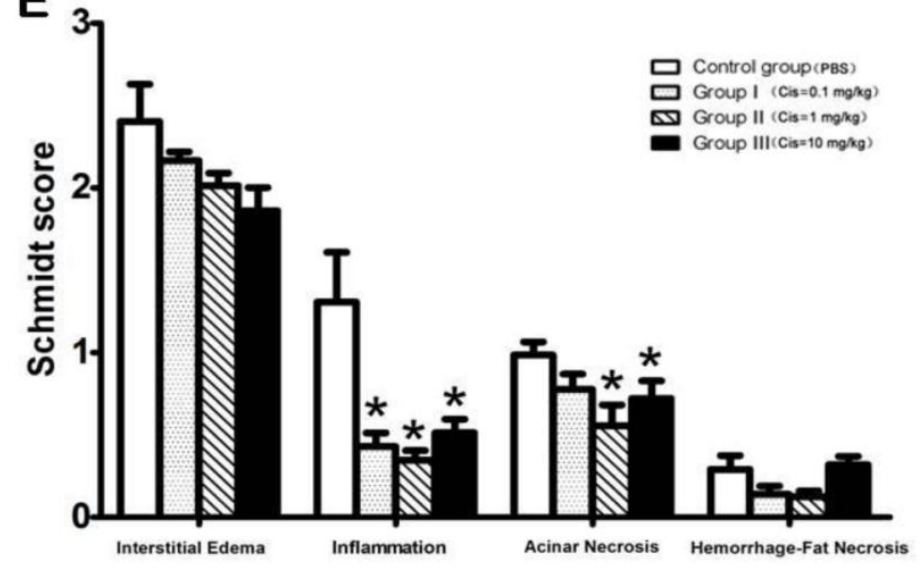

Fig. 2. Histopathology of pancreatic graft after transplantation. A) PBScontrol group. A large number of leukocytes infiltrated into graft (Black arrow) and acinar cell necrosis (Red arrow) was observed in PBS treated controls. B-D) Cis pretreated group $(B=0.1 \mathrm{mg} / \mathrm{kg}, \quad \mathrm{C}=1.0 \mathrm{mg} / \mathrm{kg}$, $D=10 \mathrm{mg} / \mathrm{kg}$ ). Note that pretreatment of $\mathrm{Cis}$ in recipient displays reduction of local inflammation. E) Histological score of PTP. Note that $\mathrm{Cis}$ significantly inhibited graft-infiltrating cells in a dose depend manner. Inhibition of acinar necrosis was also noticed. All sections harvested from each group were scored with Schmidt's method for inflammatory infiltration, edema formation by three independent pathologists [I I]. N=6 mice per group. 
A

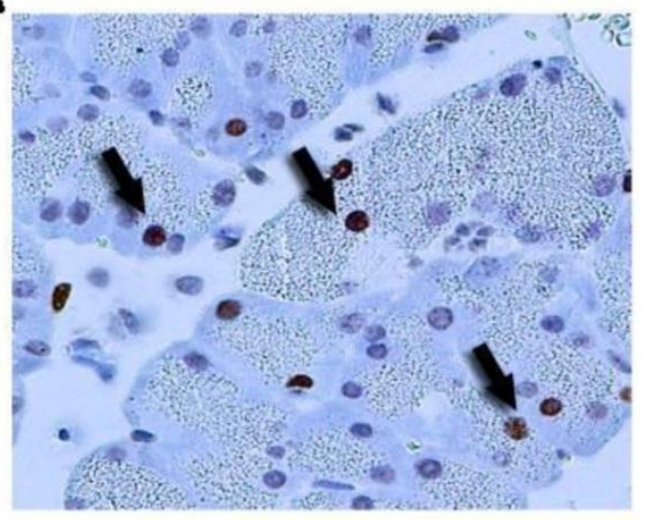

C

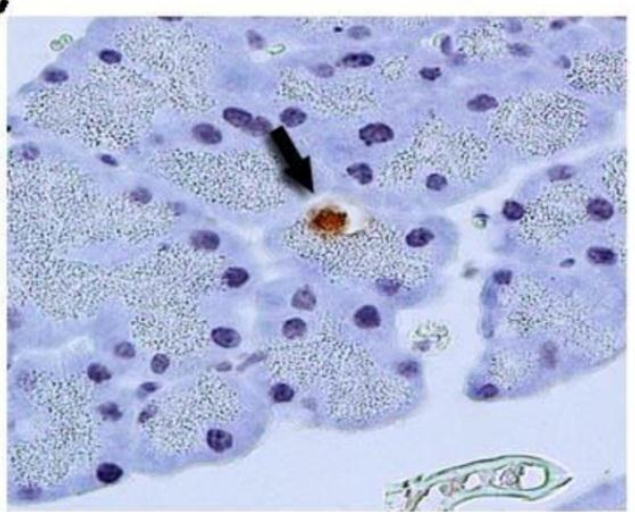

B

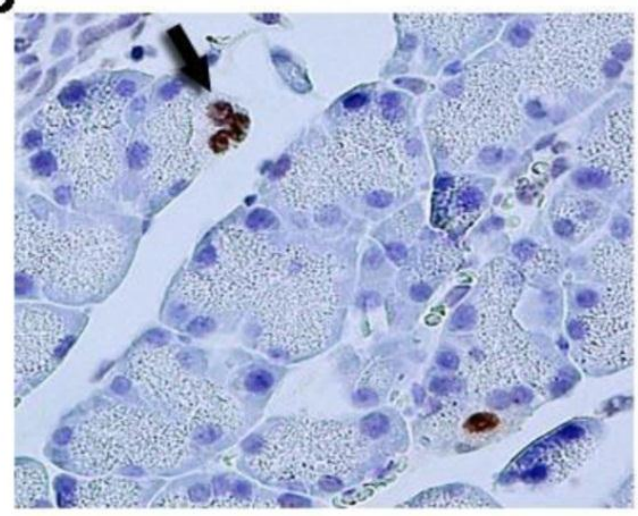

D

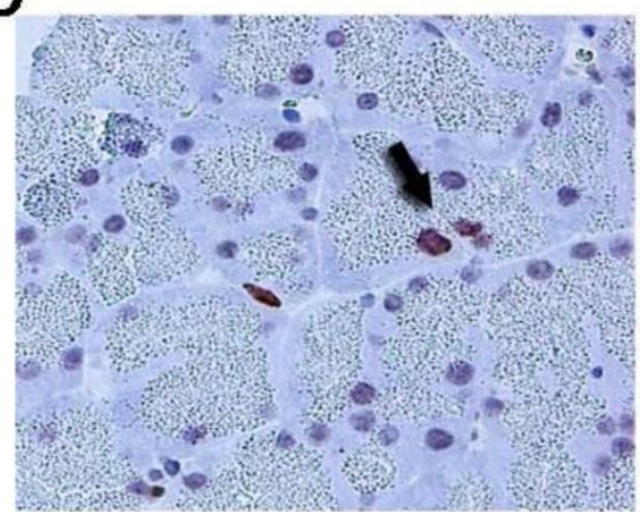

E

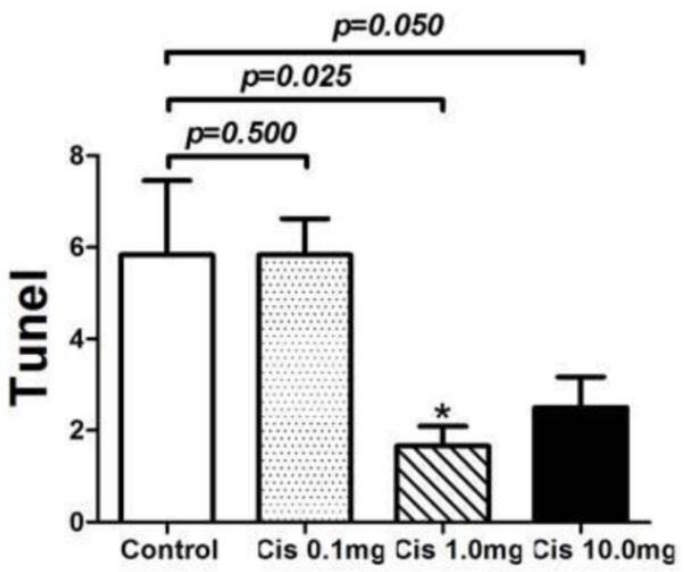

Fig. 3. Tunel assay of acinar cells after transplantation. The staining of apoptotic cells by the Tunel method in graft. A) PBS-control group. Pancreatic acinar cells apoptosis were observed in PBS-control group (Arrow). B-D) Cis pretreated group (B=0.1 mg/kg, $C=1.0 \mathrm{mg} / \mathrm{kg}, \mathrm{D}=10 \mathrm{mg} / \mathrm{kg}$ ). After pretreatment with Cis, the number of pancreatic acinar cell apoptosis (Arrow) was reduced. E) Number of apoptotic acinar cells in the graft. Note that $\mathrm{Cis}$ decreased the number of acinar cell apoptosis. Stationary of Tunel assay was conducted by quantification of apoptosis cell in 10 randomly high power fields. $\mathrm{N}=6$ mice per group. 




Fig. 4. Serum Amylase level after transplantation. Serum amylase was measured 6-hr after pancreas transplantation. Cis decreased serum amylase level at a dose of $1.0 \mathrm{mg} / \mathrm{kg}$. $\mathrm{N}=6$ mice per group.
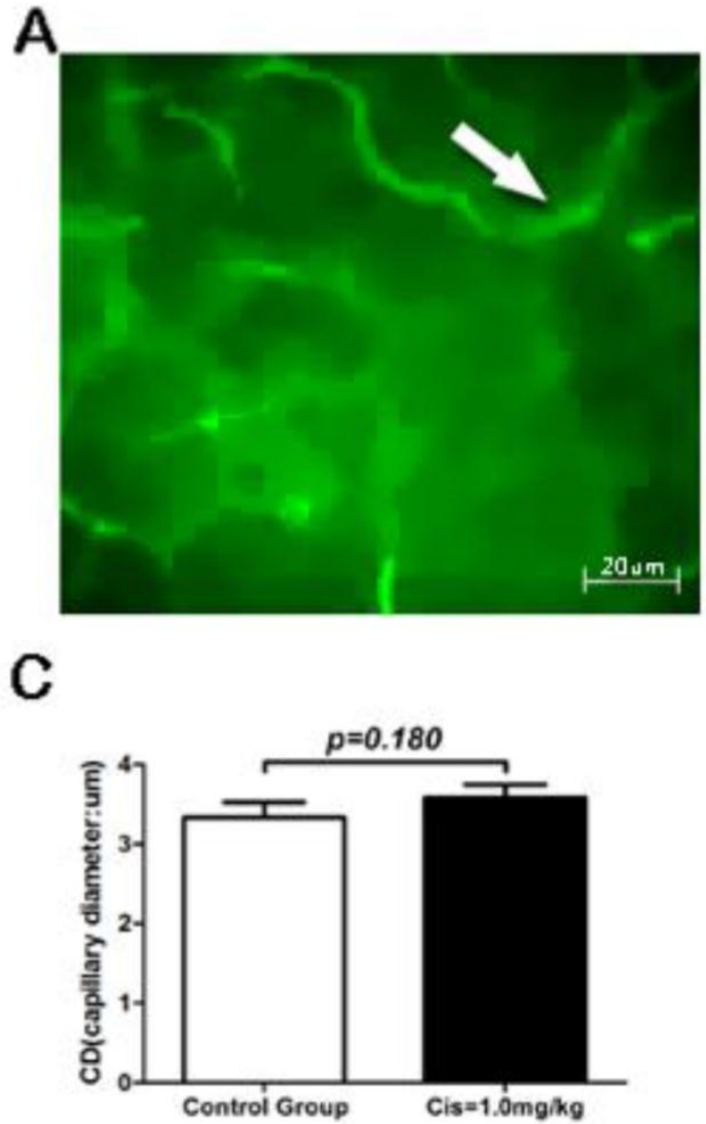

B

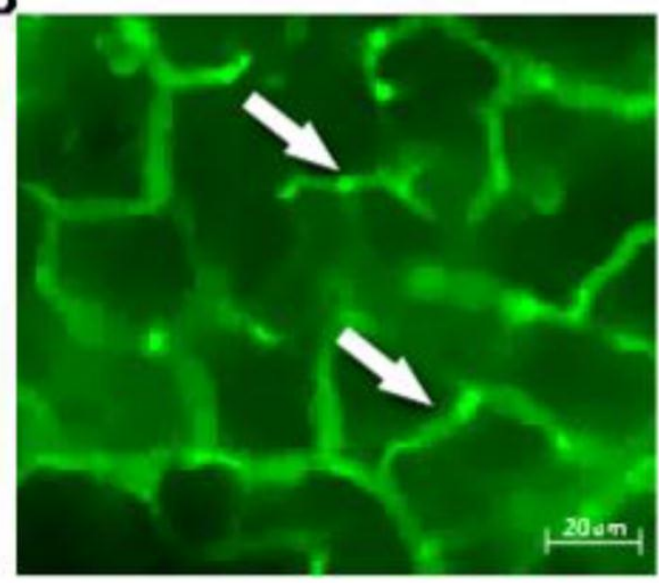

D

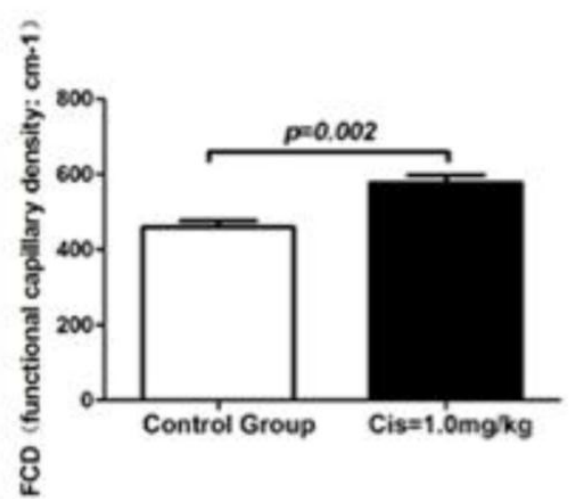

Fig. 5. Intravital fluorescence microscopy (IVM) of the graft. A) PBS-control group. IVM displayed severe perfusion dysfunction in grafts, the capillary density was significantly reduced by IR injure after transplantation. B) Cis pretreated group (I.0mg/kg). Cis pretreatment significantly improved graft microcirculation, a richer capillary network was observed (Arrow). C) Capillary diameters (CD) of pancreas exocrine tissue. D) Functional capillary density (FCD) of pancreas exocrine tissue. Note that FCD was significantly increased in Cis pretreated group. Original magnification: $350 \mathrm{X}$. N=6 mice per group, each mice collected eight time points of data. 


\section{Cis pretreatment inhibits local inflammatory cell infiltration in graft.}

We next examine neutrophil or monocytes / macrophages sequestration in the graft. We proposed that myeloperoxidase (MPO) released by these cells may play an important role in the onset of PTP. To this end, we measured MPO activity in neutrophils, monocytes/macrophages in pancreatic grafts by immunohistochemistry. The result showed that pre- treatment of Cis in recipients display less MPO positive cells compared with the PBS-transplanted pancreas (Fig. 6A-D). Semi quantitative analyses of MPO positive cells per field demonstrated that pretreatment of Cis at the doses between $0.1 \mathrm{mg} / \mathrm{kg}$ to 10 $\mathrm{mg} / \mathrm{kg}$ significantly $(\mathrm{p}=0.002)$ decreased the numbers of MPO positive cells (Fig. 6E). This result indicated that Cis significantly inhibits activity of neutrophils and monocytes.

B

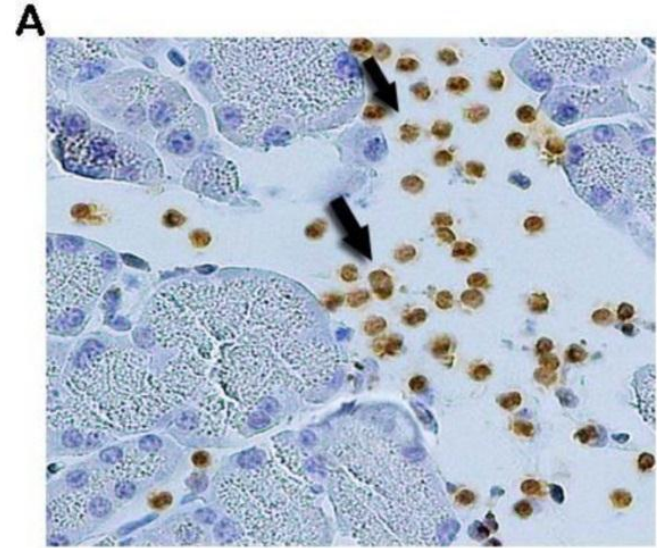

\section{C}

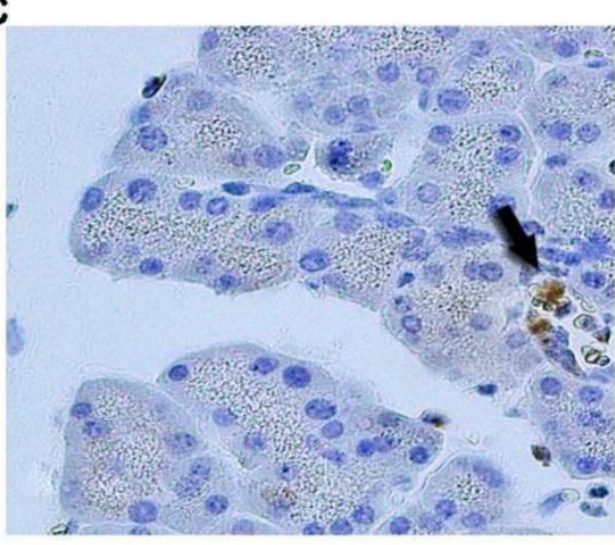

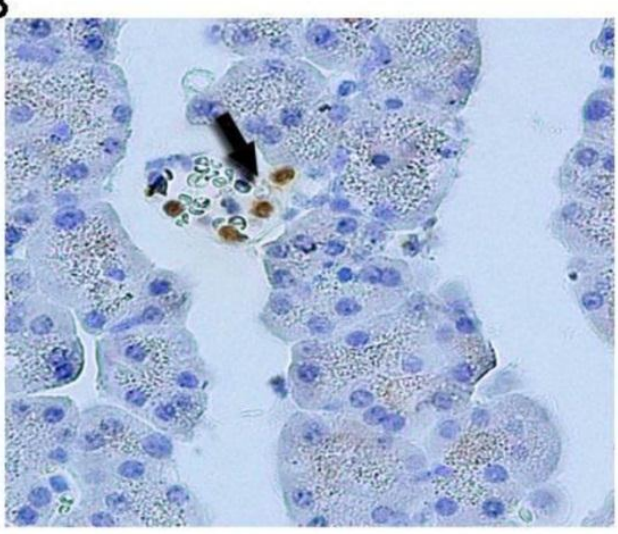

D

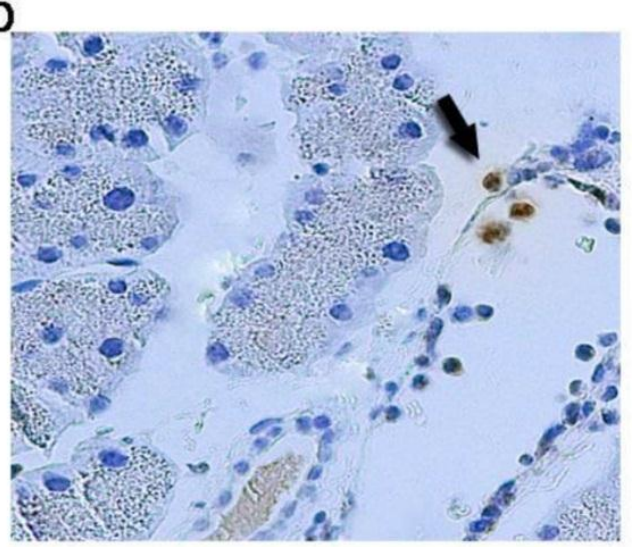

E

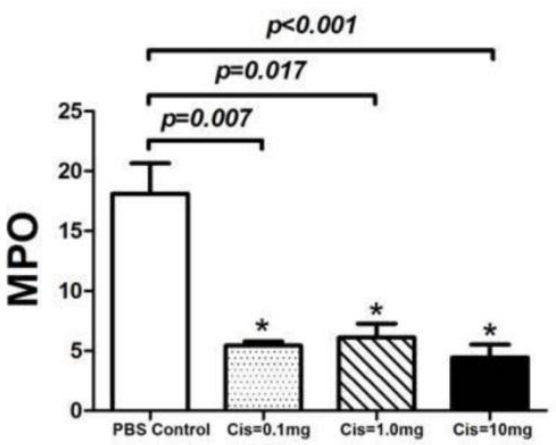

Fig. 6. Immunohistochemistry of MPO in Graft. Control group: a large number of MPO+ cells infiltrating into the acinar interstitial (Arrow). B-D) Cis pretreated groups. Pretreatment with $\mathrm{Cis}$ significantly reduced the $\mathrm{MPO}^{+}$cells in different doses $(B=0.1 \mathrm{mg} / \mathrm{kg}$, $C=1 \mathrm{mg} / \mathrm{kg}, \mathrm{D}=10.0 \mathrm{mg} / \mathrm{kg}$ ). E) Numbers of infiltrated $\mathrm{MPO}{ }^{+}$cells in the graft. Note that Cis reduced numbers of MPO ${ }^{+}$cells in pancreatic interstitial. Stationary of MPO immunohistochemistry was conducted by quantification of MPO+ cell in 10 randomly high power fields. $\mathrm{N}=6$ mice per group. 

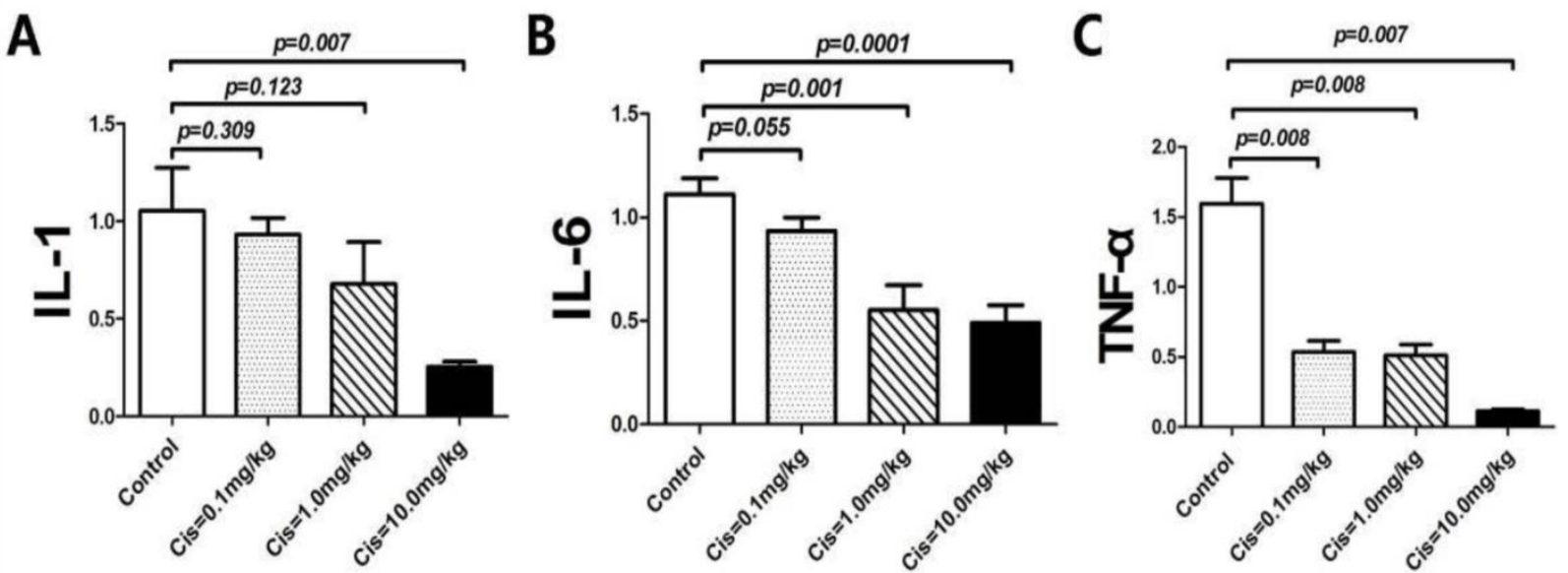

Fig. 7. Level of inflammatory cytokines in graft extracts after transplantation. A)IL-I.B) IL-6.C) TNF-alpha. Note that the expression of IL-I, IL-6 and TNF-alpha in the pancreas graft was dropped after Cis. The results were expressed as ratio of gray value of Target band and the beat-Actin bend. $\mathrm{N}=6$ mice per group.

\section{Cis inhibit the inflammatory cytokine-cascade after transplantation.}

Cascade of inflammatory cytokines is proved to be a critical step in IR injury and acute pancreatitis [16]. Using tissue extract and western blotting, we tested the tissue level of IL-1 (Fig.7A), IL-6 (Fig.7B), and TNF-alpha (Fig.7C) in pancreatic graft 6 hour after transplantation. The result showed that protein expression of IL-1, IL-6 and TNF-alpha in the grafts was inhibited by the pretreatment of Cis in recipients. While the reduction of IL-1 and TNF-alpha expression did not achieve a statistical significance at the lower doses, the suppression of IL-6 expression is in a dose-dependent manner. This variation may be due to the fate that cytokine cascade is a dynamic process, and thus a single time measurement at 6 hours may not be able to determine the dose dependent effect.

\section{Cis suppresses HMGB-I translocation and ex- pression in host inflammatory cells.}

Recent studies by Cardinal et al demonstrated that inhibition of HMGB-1 release from nuclear to cytoplasm by Cis protects liver from IR induced injury $[9,17]$. To explore the potential mechanism by which Cis attenuates PTP, we examined the intracellular localization and protein expression of HMGB-1 using immunofluorescence staining and western blotting. Immunofluorescence demonstrated that in graft infiltrating inflammatory cells from the control group with pretreatment of PBS, HMGB1 is located predominantly in cytoplasm and less in nucleus. In contrast, pretreatment of Cis in doses between $0.1 \mathrm{mg} / \mathrm{kg}$ to $10 \mathrm{mg} / \mathrm{kg}$ showed decreased cytoplasmic signal and increased nuclear location of HMGB-1.
This result implies Cis prevented IR induced shuttling of HMGB-1 from nucleus to cytoplasm (Fig. 8A-F). Next, we examined if Cis can reduce protein expression of HMGB-1. Western Blot analyses showed that pretreatment with Cis also evidently decreases the level of HMGB-1 in tissue extract of pancreas graft (Fig. 8G-H).

\section{Discussion}

PTP due to IR injury and poor preservation is a critical complication in pancreas transplantation. In the present study, we find that pretreatment of Cis in recipient mice attenuates PTP as evident by the reduction of graft inflammatory response and the protection of acinar cell apoptosis. The protective effects by Cis may involve multiple steps including reduction of inflammatory cell infiltration and activation, improvement of the microcirculation, inhibition of inflammatory cytokine-cascade and reduction of HMGB-1 expression and translocation from nucleus to cytoplasm in graft-infiltrating inflammatory cells.

Activated graft-infiltrating granulocytes and monocytes/macrophages release various cytokines and chemokines, which may cause pancreatic interstitial edema, microcirculation dysfunction, acinar cells apoptosis/ necrosis, and ultimately lead to a severe PTP [18]. In our model, we used a modified non-touch technique, based on Masayuki tori's description [6], which preserved exocrine drainage. We believe this mending the shortcoming from an occlusion of pancreatic duct that leads to additional damage of tissue. As a result, our model showed less necrosis and hemorrhage after reperfusion, which corresponds the clinical transplants well [19]. 

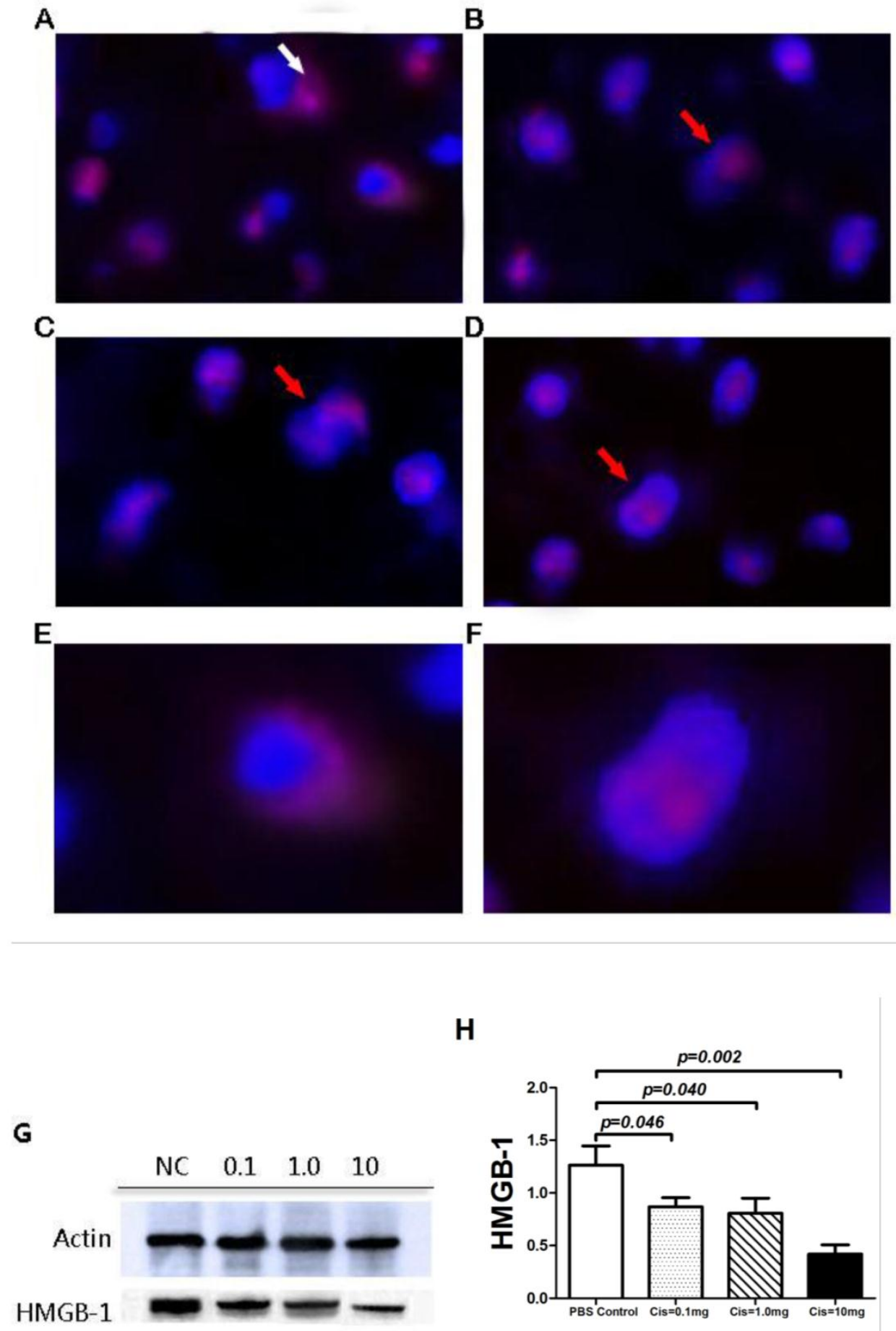

Fig. 8. Intracellular staining and protein expression of HMGB-I in graft. HMGB-I(Cy3-Red), cell nuclear (DAPI-Blue). A) Control group. Note that HMGB-I is observed in cytoplasm (White arrow). B-D) Cis pretreatment group $(B=0.1 \mathrm{mg} / \mathrm{kg}, C=1 \mathrm{mg} / \mathrm{kg}$ and, $D=10 \mathrm{mg} / \mathrm{kg}$ ). Note that majority of HMGB-I is located nuclear (Red Arrow) E) HMGB-I release from nuclear of graft-infiltrating cell in Control group. F) HMGB-I was retained in cell nuclear by pretreatment of Cis. G-H) Protein expression of HMGB-I.The protein expression of HMGB-I in graft level was significantly reduced by the pretreatment of Cis. The results were expressed as ratio of gray value of target band and the beat-Actin bend. $\mathrm{N}=6$ mice per group.

Microcirculatory dysfunction had been considered to be an indicator for the severity of pancreatitis[20]. Several studies have used FCD as an indicator of the quality of tissue perfusion in various animal models [5, 21-25]. FCD is considered to be a sensitive real-time indicator, which enable to reflect the performance of functional perfusion of the tissue [26]. Studies by Nolte et al have shown that FCD is able to 
yield highly reproducible, user-independent results under physiologic conditions and the pathophysiologic conditions of ischemia-reperfusion in various pancreas models [27]. Using FCD as the parameter for microcirculation, we showed that Cis may inhibit the occurrence of perfusion disorders during the early period of PTP. We postulated that the improvement of microcirculation evidenced by FCD may be due to the reduction of the inflammatory cell infiltration by Cis.

MPO and the cascade release of inflammatory cytokines including TNF-alpha, IL-1, and IL-6 are important mediators of inflammation which play critical role in the pathogenesis of PTP. Our study has demonstrated that Cis not only inhibit inflammatory cell infiltration, but also suppress their activation by the release of MPO and inflammatory cytokines.

It is believed that the release of HMGB-1 from nucleus is a trigger for the cascades of activation of the inflammation [28, 29]. Studies by Tsung et al showed that Cis treatment benefits to the prevention of hepatic IR injury by reducing the inflammatory cell infiltration and the production of inflammatory mediators [30, 31]. Indeed, the release of HMGB-1 was identified as an early event in the pathogenesis of organ IR impairments, followed by cytokine cascade and mononuclear infiltration [28-30]. Recent studies had shown elevation of HMGB-1 in a mice model of Caerulein induced acute pancreatitis, and inhibition of HMGB-1 attenuated the severity of the pancreatitis [30]. In our study, the inhibition of translocation of HMGB-1 from nucleus to cytoplasm was consistent with the recent report that Cis inhibits HMGB-1 releasing from nucleus of mononuclear cell in IR liver [31-33]. However the fate that we observed the reduction of HMGB-1 in graft extract of Cis treated groups was unexpected. This might be at least in part due to the reduction of the numbers of inflammatory cells infiltration into the graft. Whether Cis is able to suppress protein expression of HMGB-1 is subjected to further investigation.

Cis as general chemotherapeutic agent has been used in a range of doses from pharmacological to toxicological levels depending on their applications. Previous study showed that $\mathrm{Cis}$ at doses up to $10 \mathrm{mg} / \mathrm{kg}$ did not alter liver or kidney function compared to normal saline solution [9]. On the other hand, other studies have shown that Cis at a single dose of $10 \mathrm{mg} / \mathrm{kg}$ is clearly nephrotoxic and hepatotoxic. In our study, a relatively higher dose of Cis $(10 \mathrm{mg} / \mathrm{kg})$ appears not lead to a better treatment outcome.

While our study is able to show the protective effect of PTP at a dose of $1 \mathrm{mg} / \mathrm{kg}$, we were also unable to show a dose dependent effect for some of the assays. This may be due to the sensitivity of methods used and the systemic toxic effects of Cis at high doses in the individual recipient mice. It is also possible due to the fate that a single time point of 6 hours study after reperfusion is not sufficient to monitor the entire development of IR induced PTP. While we showed the evidence that pretreatment of Cis in recipients is able to attenuate graft pancreatitis by the inhibition of inflammation, these limitations in the study will further promote us to clarify the mechanism of Cis action in vitro.

\section{Acknowledgments}

The authors would like to thank Prof. Xiaowen Feng and for pathology guide. The work is supported in part by Team Program of Science and Technology Bureau of Zhejiang Province (No.2009R50038), and Zhejiang Provincial Natural Science Foundation (No.J20100398).

\section{Abbreviation}

CIS: Cisplatin; PTP: Post-Transplantation Pancreatitis; HMGB-1: High-Motility Group Box protein-1; IVM: Intravital fluorescence microscopy; IR: Ischemia reperfusion; MPO: myeloperoxidase.

\section{Conflict of Interests}

The authors have declared that no conflict of interest exists.

\section{References}

1. Lillehei RC, Idezuki Y, Feemster JA, Dietzman RH, Kelly WD, Merkel FK, et al. Transplantation of stomach, intestine, and pancreas: experimental and clinical observations. Surgery. 1967; 62: 721-41.

2. Kelly WD, Lillehei RC, Merkel FK, Idezuki Y, Goetz FC. Allotransplantation of the pancreas and duodenum along with the kidney in diabetic nephropathy. Surgery. 1967; 61: 827-37.

3. Perosa M, Boggi U, Cantarovich D, Robertson P. Pancreas transplantation outside the USA: an update. Current opinion in organ transplantation. 2011; 16:135-141.

4. Lodhi SA, Lamb KE, Meier-Kriesche HU. Solid organ allograft survival improvement in the United States: the long-term does not mirror the dramatic short-term success. Am J Transplant. 2011; 11: 1226-35.

5. Woeste G, Wullstein C, Meyer S, Usadel KH, Hopt UT, Bechstein $\mathrm{WO}$, et al. Octreotide attenuates impaired microcirculation in postischemic pancreatitis when administered before induction of ischemia. Transplantation. 2008; 86: 961-7.

6. Maglione M, Hermann M, Hengster P, Schneeberger S, Mark $\mathrm{W}$, Obrist $\mathrm{P}$, et al. A novel technique for heterotopic vascularized pancreas transplantation in mice to assess ischemia reperfusion injury and graft pancreatitis. Surgery. 2007; 141: 682-9.

7. Hackert T, Werner J, Uhl W, Gebhard MM, Buchler MW, Schmidt J. Reduction of ischemia/reperfusion injury by antithrombin III after experimental pancreas transplantation. Am J Surg. 2005; 189: 92-7.

8. Serr F, Lauer H, Armann B, Ludwig S, Thiery J, Fiedler M, et al. Sirolimus improves early microcirculation, but impairs regen- 
eration after pancreatic ischemia-reperfusion injury. Am J Transplant. 2007; 7: 48-56.

9. Cardinal J, Pan P, Dhupar R, Ross M, Nakao A, Lotze M, et al. Cisplatin prevents high mobility group box 1 release and is protective in a murine model of hepatic ischemia/reperfusion injury. Hepatology. 2009; 50: 565-74.

10. Liu XY, Xue L, Zheng X, Yan S, Zheng SS. Pancreas transplantation in the mouse. Hepatobiliary \& pancreatic diseases international : HBPD INT. 2010; 9: 254-8.

11. Schmidt J, Rattner DW, Lewandrowski K, Compton CC, Mandavilli U, Knoefel WT, et al. A better model of acute pancreatitis for evaluating therapy. Annals of surgery. 1992; 215: 44-56.

12. Liu YX, Jin LM, Zhou L, Xie HY, Jiang GP, Wang Y, et al. Mycophenolate mofetil attenuates liver ischemia/reperfusion injury in rats. Transpl Int. 2009; 22: 747-56.

13. Maglione M, Oberhuber R, Cardini B, Watschinger K, Hermann $\mathrm{M}$, Obrist $\mathrm{P}$, et al. Donor pretreatment with tetrahydrobiopterin saves pancreatic isografts from ischemia reperfusion injury in a mouse model. Am J Transplant. 2010; 10: 2231-40.

14. Maglione M, Hermann M, Hengster P, Schneeberger S, Mark $\mathrm{W}$, Obrist $\mathrm{P}$, et al. Tetrahydrobiopterin attenuates microvascular reperfusion injury following murine pancreas transplantation. Am J Transplant. 2006; 6: 1551-9.

15. Katarı́na Vajdova', Stefan Heinrich, Yinghua Tian, Rolf Graf, and Pierre-Alain Clavien. Ischemic Preconditioning and Intermittent Clamping Improve Murine Hepatic Microcirculation and Kupffer Cell Function after Ischemic Injury. Liver Transplantation. 2004; 10( 4): 520-528.

16. Tang D, Kang R, Zeh HJ, 3rd, Lotze MT. High-mobility group box 1 , oxidative stress, and disease. Antioxidants \& redox signaling. 2011; 14: 1315-35.

17. Allan Tsung,Rohit Sahai,Hiroyuki Tanaka,Atsunori Nakao,Mitchell P. Fink,Michael T. Lotze,Huan Yang,Jianhua Li,Kevin J. Tracey,David A. Geller,Timothy R. Billiar.The nuclear factor HMGB1 mediates hepatic injury after murine liver ischemia-reperfusion. JEM. 2005; 201:1135-1143.

18. Oberhuber, R.; Maglione, M.; Cardini, B.; Hermann, M.; Obrist, P.; Schneeberger, S.; Margreiter, R.; Pratschke, J.; Werner, E.; Brandacher, G. Tetrahydrobiopterin Administration Prevents Lethal Graft Pancreatitis in A Murine Pancreas Transplantation Model: 2974. Transplantation. 2010; 90: 983.

19. JonCardinal,Pinhua Pan,Rajeev Dhupar,Mark Ross,Atsunori Nakao,Michael Lotze,Timothy Billiar,David Geller,Allan Tsung. Cisplatin prevents high mobility group box 1 release and is protective in a murine model of hepatic ischemia/reperfusion injury. Hepatology. 2009; 50:565-574.

20. Cuthbertson CM, Christophi C. Disturbances of the microcirculation in acute pancreatitis. Br J Surg. 2006; 93: 518-30.

21. Sorg H, Lorch B, Jaster R, Fitzner B, Ibrahim S, Holzhueter SA, et al. Early rise in inflammation and microcirculatory disorder determine the development of autoimmune pancreatitis in the MRL/Mp-mouse. Am J Physiol Gastrointest Liver Physiol. 2008; 295: G1274-80.

22. Preissler G, Massberg S, Waldner H, Messmer K. Intermittent capillary perfusion in rat pancreas grafts following short- and long-term preservation in University of Wisconsin solution. Transpl Int. 2006; 19: 325-32.

23. Obermaier R, von Dobschuetz E, Muhs O, Keck T, Drognitz O, Jonas $\mathrm{L}$, et al. Influence of nitric oxide on microcirculation in pancreatic ischemia/reperfusion injury: an intravital microscopic study. Transpl Int. 2004; 17: 208-14.

24. V. Schmitza, K.-D. Schaserb, P. Olschewskia, P. Neuhausa, G. Puhla. In vivo Visualization of Early Microcirculatory Changes following Ischemia/Reperfusion Injury in Human Kidney Transplantation. Eur Surg Res 2008;40:19-25.

25. Pedro Cabrales, Amy G. Tsai and Marcos Intaglietta. Microvascular pressure and functional capillary density in extreme hemodilution with low- and high-viscosity dextran and a low-viscosity $\mathrm{Hb}$-based $\mathrm{O}$ carrier. Am J Physiol Heart Circ Physiol. 2004; 287:H363-H373.

26. Keck T, Friebe V, Warshaw AL, Antoniu BA, Waneck G, Benz S, Hopt UT, Fernández-del-Castillo C. Pancreatic proteases in serum induce leukocyte-endothelial adhesion and pancreatic microcirculatory failure. Pancreatology. 2005;5(2-3):241-50.

27. Nolte D, Zeintl H, Steinbauer M, Pickelmann S, Messmer K,et al. Functional capillary density: an indicator of tissue perfusion. International Journal of Microcirculation. 1995, 15(5):244-9.

28. Liu A, Dirsch O, Fang H, Sun J, Jin H, Dong W, et al. HMGB1 in ischemic and non-ischemic liver after selective warm ischemia/reperfusion in rat. Histochemistry and cell biology. 2011; 135: $443-52$

29. Wu H, Ma J, Wang P, Corpuz TM, Panchapakesan U, Wyburn $\mathrm{KR}$, et al. HMGB1 contributes to kidney ischemia reperfusion injury. Journal of the American Society of Nephrology: JASN. 2010; 21: 1878-90.

30. Yuan H, Jin X, Sun J, Li F, Feng Q, Zhang C, et al. Protective effect of HMGB1 a box on organ injury of acute pancreatitis in mice. Pancreas. 2009; 38: 143-8.

31. Jon S. Cardinal, Pinhua Pan, Rajeev Dhupar, John Klune, Sung Cho, David A. Geller, Allan Tsung. Cisplatin retains high-mobility group box 1 (HMGB1) intranuclearly and protects against hepatic ischemia reperfusion (I/R) injury. Journal of the American College of Surgeons. 2008;207: S21-S22.

32. Pinhua Pan,Jon Cardinal,Rajeev Dhupar,Matthew R. Rosengart,Michael T. Lotze,David A. Geller,* Timothy R. Billiar,Allan Tsung,Low-dose cisplatin administration in murine cecal ligation and puncture prevents the systemic release of HMGB1 and attenuates lethality. Journal of Leukocyte Biology.2009;86: 3625-3632.

33. Jon Cardinal,Pinhua Pan,Allan Tsung. Protective role of cisplatin in ischemic liver injury through induction of autophagy. Autophagy.2009;5: 1211-1212. 